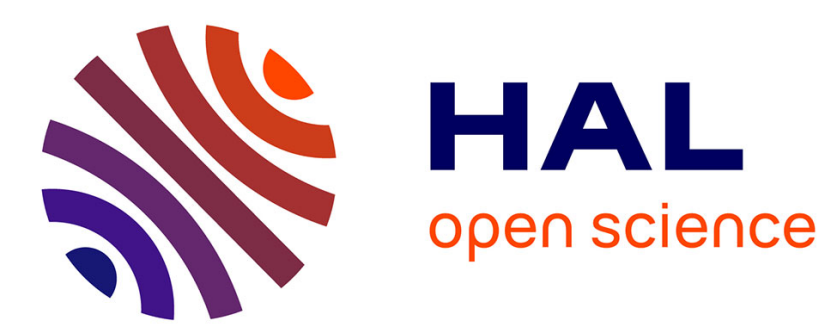

\title{
Spectrum Coordination in Energy-Efficient Cognitive Radio Networks
}

\author{
Majed Haddad, Yezekael Hayel, Oussama Habachi
}

\section{To cite this version:}

Majed Haddad, Yezekael Hayel, Oussama Habachi. Spectrum Coordination in Energy-Efficient Cognitive Radio Networks. IEEE Transactions on Vehicular Technology, 2015, 64 (5), pp.2112-2122. 10.1109/TVT.2014.2339271. hal-03484689

\section{HAL Id: hal-03484689 \\ https://hal.science/hal-03484689}

Submitted on 17 Dec 2021

HAL is a multi-disciplinary open access archive for the deposit and dissemination of scientific research documents, whether they are published or not. The documents may come from teaching and research institutions in France or abroad, or from public or private research centers.
L'archive ouverte pluridisciplinaire HAL, est destinée au dépôt et à la diffusion de documents scientifiques de niveau recherche, publiés ou non, émanant des établissements d'enseignement et de recherche français ou étrangers, des laboratoires publics ou privés. 


\title{
Spectrum Coordination and Learning in Energy Efficient Cognitive Radio Networks
}

\author{
Yezekael Hayel$^{*}$, Majed Haddad ${ }^{\dagger}$ and Oussama Habachi ${ }^{\dagger}$ \\ * CERI/LIA, University of Avignon, Agroparc BP 1228, Avignon, France \\ ${ }^{\dagger}$ INRIA, Sophia Antipolis, France
}

\begin{abstract}
In this paper, we propose an algorithmic perspective of the Stackelberg game model introduced in [1] applied to cognitive radio networks (CRN). Typically, we assume that individual users attempt to access to the wireless spectrum while maximizing their individual energy efficiency. Having looked at the main properties of the proposed energy efficient and in particular the one related to spectrum coordination, we address the problem of sensing. Then, we provide a deep algorithmic analysis on how primary and secondary users can reach such a spectrum coordination using an appropriate learning process. We validate our results through extensive simulations and compare the proposed algorithm to some typical scenarios including the non-cooperative case in [2] and the throughput-based-utility systems. Specifically it is shown that the proposed Stackelberg decision approach maximizes the energy efficiency while still optimizing the throughput at the equilibrium.
\end{abstract}

\section{INTRODUCTION}

Cognitive radio technology has been proposed first to increase the throughput of mobiles for the next generation of wireless technologies. This enhancement is possible with an efficient use of the wireless spectrum and specifically spectrum holes. Indeed, primary users that have a specific and licensed access to the spectrum let part of the spectrum unused in different time and geographic location. Many works have been done for optimizing the behavior of secondary users in cognitive radio networks, see [3] for a survey. However, most of previous works have focused on spectrum sharing [4] or cognitive radio networks and interference avoidance [5]. Consequently, the energy efficiency aspect in this setting was largely ignored. Green communications are attracting growing attention due to various economical and environmental reasons. This has led research community to focus more to reduce energy consumption by introducing enhanced networking technologies. Motivated by the facts that mobile terminals have a limited battery life inspite of the transmission rate, green networking have spurred great interest and excitement these recent years. In the literature, energy-efficient power control game has been first proposed by Goodman et al. in [6] for flat fading channels and recently re-used by [2] for multi-carrier CDMA (code division multiple access) systems and linear receivers. Most of those works do not consider the cognitive radio technology, and therefore the capabilities of the secondary users.

The organization of the paper is the following. First, we introduce, in Section II, the CRN context and the different decision makers of the system. In section III, we give fundamental results on the existence and uniqueness of the Stackelberg equilibrium for both the primary and the secondary users. In Section III-C, we address the important property of spectrum coordination. Section IV provides a deep analysis of a learning algorithm that converges to the Stackelberg equilibrium and Section V investigates the sensing issue. Section VI illustrates some numerical results, and Section VII concludes the paper.

\section{Cognitive Radio System Model}

We consider a cognitive network composed of one primary user (leader - indexed by 1 ), having the priority to access the medium, and a secondary user (follower - indexed by 2) that accesses the medium after sensing the environment. Transmissions are slotted for both the primary user (PU) and the secondary user (SU) where each user enjoys two carriers. Although its simplicity, this scheme allows us to address the problem of hierarchically allocating transmit power in multi-carrier systems and gain insights into how to design power control strategies in a multi-carrier environment. We further assume that users transmit their data over block Rayleigh flat fading channels $g_{n}^{k}$ where $n$ stands for user $n$ and $k$ stands for the carrier. Receivers know on each block the channel gains (coherent communication assumption) whereas each transmitter has only access to the knowledge of his own channel. The signal of user $n$ is transmitted over carrier $k$ with power $p_{n}^{k}$ and the additive Gaussian noise at the receiver is i.i.d circularly 
symmetric of variance $\sigma^{2}$. For any user $n \in\{1,2\}$ the received signal-to-noise plus interference ratio (SINR) over carrier $k$ is expressed as

$$
\gamma_{n}^{k}=\frac{g_{n}^{k} p_{n}^{k}}{\sigma^{2}+\sum_{\substack{m=1 \\ m \neq n}}^{M} g_{m}^{k} p_{m}^{k}}:=p_{n}^{k} \widehat{h}_{n}^{k}
$$

It follows from the above SINR expression that the strategy chosen by a user affects the performance of other users in the network through multiple-access interference.

\section{NETWORK ENERGY EFFICIENCY ANALYSIS}

Our system model is based on the seminal paper [6] that defines the energy efficiency framework. SINR is a critical parameter for the quality of service (QoS) of the signal transmission, as it directly determines the bit error rate, which is closely related to the data throughput (average rate of successful packet delivery). In brief, when SINR is very low, data transmission results in massive errors and the throughput tends to 0 ; when SINR is very high, data transmission becomes error-free and the throughput grows asymptotically to a constant. However, achieving a high SINR level requires the user terminal to transmit at a high power, which in turn results in low battery life. This phenomenon can be concisely captured by an increasing, continuous and Sshaped "efficiency" function $f(\cdot)$, which measures the packet success rate. $R_{n}$ is the transmission rate of user $n$ over carrier $k$. We also require that to ensure that the utility is equal to zero when $p_{n}^{k}=0$. On the other hand, increasing the transmit power clearly favors the packet success rate and therefore the throughput. However, as the packet success rate tends to one, further increasing the power can lead to marginal gains in terms of throughput regarding the amount of extra power used. The following utility function allows one to measure the corresponding tradeoff between the transmission benefit (total throughput over both carriers) and cost (total power over both carriers):

$$
u_{n}\left(\mathbf{p}_{\mathbf{1}}, \mathbf{p}_{\mathbf{2}}\right)=\frac{R_{n}\left(f\left(\gamma_{n}^{1}\right)+f\left(\gamma_{n}^{2}\right)\right)}{p_{n}^{1}+p_{n}^{2}} .
$$

In this work, we consider a Stackelberg game framework in which the primary user decides first his power allocation vector $\mathbf{p}_{\mathbf{1}}=\left(p_{1}^{1}, p_{1}^{2}\right)$ and based on this value, the secondary user will adapt his power allocation vector $\mathbf{p}_{\mathbf{2}}=\left(p_{2}^{1}, p_{2}^{2}\right)$.

A Stackelberg equilibrium can be determined using a bi-level approach. First, given the action of the primary user, we compute the best-response function of the secondary user (the function $\bar{p}_{2}(\cdot)$ ), i.e. the action of the secondary user which maximizes his utility given the action of the primary user. In [1], we characterized the power allocation vector for both the primary and the secondary user. For making this paper sufficiently selfcontained, we review here the latter results.

\section{A. The secondary user's power allocation vector}

Proposition 1. Given the power allocation vector $\mathbf{p}_{\mathbf{1}}$ of the primary user, the best-response of the secondary user is given by

$$
\bar{p}_{2}^{k}\left(\mathbf{p}_{\mathbf{1}}\right)=\left\{\begin{array}{l}
\frac{\gamma^{*}\left(\sigma^{2}+g_{1}^{k} p_{1}^{k}\right)}{g_{2}^{k}}, \text { for } k=L_{2}\left(\mathbf{p}_{\mathbf{1}}\right), \\
0, \quad \text { for all } k \neq L_{2}\left(\mathbf{p}_{\mathbf{1}}\right)
\end{array}\right.
$$

with $L_{2}\left(\mathbf{p}_{\mathbf{1}}\right)=\arg \max \widehat{h}_{2}^{k}\left(p_{1}^{k}\right)$ and $\gamma^{*}$ is the unique (positive) solution of the first order equation

$$
x f^{\prime}(x)=f(x)
$$

Equation (4) has a unique solution if the efficiency function $f(\cdot)$ is sigmoidal [7], and we will use this assumption throughout our paper. This proposition says that the best-response of the secondary user is to use only one carrier, the one such that the effective channel gain $\widehat{h}_{2}^{k}$ is the best.

\section{B. The primary user's power allocation vector}

Let us now look at the optimal allocation for the primary user knowing the best-response of the secondary user. Let $\widetilde{k}$ denote the "best" carrier of the primary user, i.e. $\widetilde{k}=\arg \max _{k} g_{1}^{k}$.

Proposition 2. At the Stackelberg equilibrium, the power allocation vector $\widetilde{\mathbf{p}}_{\mathbf{1}}$ for which the primary user's utility is maximized is unique and is given by

$$
\tilde{p}_{1}^{k}=\left\{\begin{array}{l}
\frac{\sigma^{2} \gamma^{*}}{g_{1}^{k}}, \quad \text { for } \quad k=\widetilde{k}, \\
0, \quad \text { for all } \quad k \neq \widetilde{k}
\end{array}\right.
$$

where $\gamma^{*}$ is the unique (positive) solution of the first order Equation (4).

Proposition 2 says that the utility of primary user is maximized when it transmits only over its best carrier. As a result, we observe that the carrier which do not contribute enough energy efficiency to outweigh the interference degradation caused by the secondary user's transmission is switched "off". 


\section{Spectrum coordination}

Prop. 1 and Prop. 2 suggest that both the secondary user and the primary user transmit on only one carrier depending on their channel gains. The next proposition claims that introducing a certain degree of hierarchy in a multi-carrier system induces a natural coordination pattern where users have incentive to choose their transmitting carriers in such a way that they always transmit on orthogonal channels.

Proposition 3. Introducing hierarchy between users in a multi-carrier energy efficient power control game "pushes" users to coordinate their actions in such a way that they always transmit on different carriers.

\section{LEARNING-BASED APPROACH}

Determining the equilibrium strategy of both the primary and the secondary user requires in practice the knowledge of several information that can not be observed in a realistic scenario. We propose, in this section, an on-policy learning-based algorithm that allow the primary user and the secondary user to determine their strategies on-the-fly. As we can see in Algorithm 1 , the PU maintains the state-value function $q(\mathbf{g}, \mathbf{p})$ as a lookup table, which determines the optimal action to chooses in the current time slot. $q(\mathbf{g}, \mathbf{p})$ is updated as follows:

$$
\begin{aligned}
q\left(\mathbf{g}^{t-1}, \mathbf{p}^{t-1}\right) \leftarrow & \beta_{t} q\left(\mathbf{g}^{t-1}, \mathbf{p}^{t-1}\right) \\
& +\left(1-\beta_{t}\right)\left(u_{1}+\gamma q\left(\mathbf{g}^{t}, \mathbf{p}^{t}\right)\right),
\end{aligned}
$$

where $\beta_{t}$ is a learning rate factor satisfying $\sum_{t=1}^{\infty} \beta_{t}=$ $\infty, \sum_{t=1}^{\infty}\left(\beta_{t}\right)^{2}<\infty$, e.g., $\beta_{t}=\frac{1}{t}$.

The SU chooses its action following state-value function $Q(\mathbf{g}, \mathbf{p})$, which is updated as follows:

$$
\begin{aligned}
Q\left(\mathbf{g}^{t-1}, \mathbf{p}^{t-1}\right) \leftarrow & \alpha_{t} Q\left(\mathbf{g}^{t-1}, \mathbf{p}^{t-1}\right) \\
& +\left(1-\alpha_{t}\right)\left(u_{2}+\gamma Q\left(\mathbf{g}^{t}, \mathbf{p}^{t}\right)\right),
\end{aligned}
$$

where $\alpha_{t}$ is a learning rate factor satisfying $\sum_{t=1}^{\infty} \alpha_{t}=$ $\infty, \sum_{t=1}^{\infty}\left(\alpha_{t}\right)^{2}<\infty, e . g ., \beta_{t}=\frac{1}{t}$.

The pseudo-code for the proposed algorithm is given in Algorithm 1.

\section{Spectrum Sensing}

In the current Stackelberg model, Prop. 3 claims that, the SU transmits over a certain frequency carrier in order to reach SINR $\gamma^{*}$ (solution of the unique (positive) solution of the first order Equation (4)) only when the primary user does not. This enables public access to the new spectral ranges without sacrificing the transmission quality of the actual license owners. Typically,

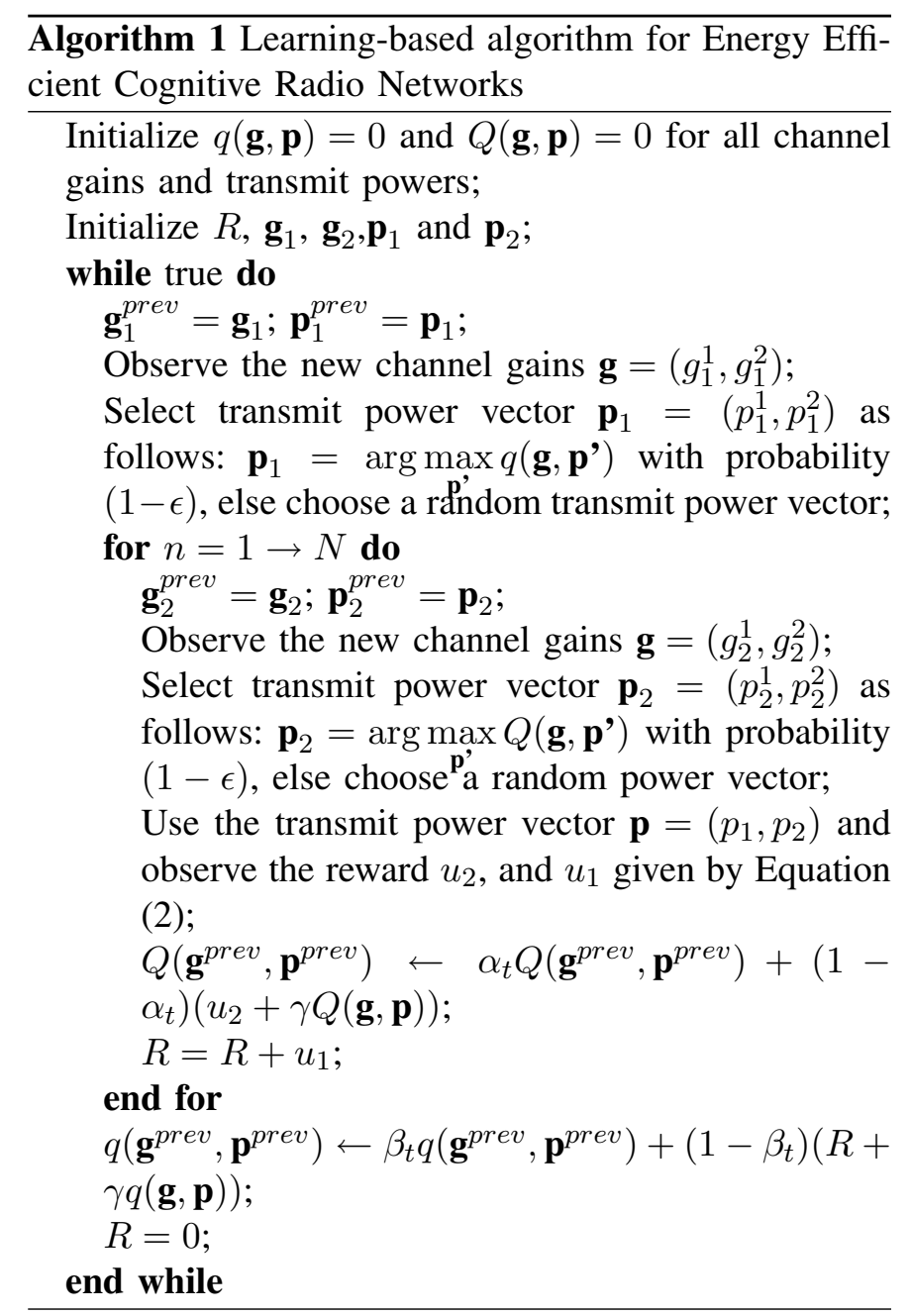

the primary user comes first in the system, estimates his channel gains $\left(g_{1}^{k}\right)$ over all carriers and adapts his transmit power using Prop. 2. The secondary user comes in the system randomly and estimates his channel links $\left(g_{2}^{k}\right)$ over all carriers. Such an assumption could be further justified by the fact that in an asynchronous context, the probability that two users decide to transmit at the same moment is negligible as the number of users is limited. Thus, within this setting, the primary user is assumed to be oblivious to the presence of the secondary user. The PU communicates with his BS while the SU listens to the wireless channel. The SU has only to reliably detect the carrier used by the PU and not the PU's transmit power as it is the case in the single carrier context in [8]). Then, he adapts his transmit power to fill detected voids in the spectrum using Prop. 1 in an overlay fashion. Many techniques were developed in order to detect the holes in the spectrum band (energy detection [9], feature detection [10], etc ). 


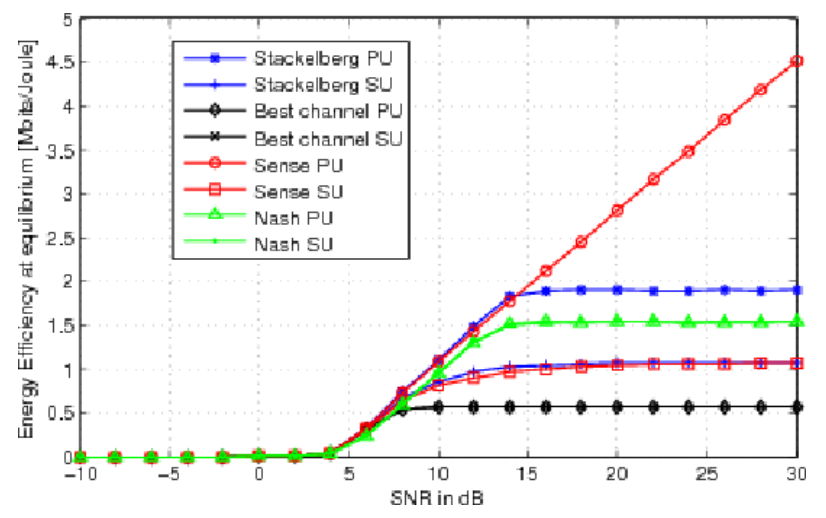

Fig. 1. Energy efficiency at the equilibrium as function of the SNR for different schemes.

\section{NUMERICAL ILLUSTRATION}

In this section, we present a comprehensive Matlabbased simulation of the $\mathrm{CRN}$ described in the previous sections. We consider the energy efficiency function proposed in most papers dealing with power allocation games that is $f(x)=\left(1-e^{-x}\right)^{M}$, where $M=100$ is the block length in bits. 10000 scenarios are simulated to remove the random effects from Rayleigh fading. $\mathrm{SNR}=1 / \sigma^{2}$ and the rate $R=1 \mathrm{Mbps}$ for both the PU and the SU.

\section{A. Energy Efficiency as function of the SNR}

This section is devoted to performance comparison of the proposed Stackelberg scheme with respect to traditional schemes. As far as sum energy efficiency comparison is concerned, this can be conducted by considering the four following schemes:

- the Stackelberg model: the one proposed in this contribution,

- the Nash model: each user chooses his power level according to [2],

- the best channel model: each user chooses to transmit on his "best" channel (i.e., the one with the best channel gain) without sensing,

- the best channel with sensing: the primary user chooses the "best" channel to transmit on. The secondary user senses the spectrum and transmits on the vacant sub-band. Here we assume perfect sensing of the idle sub-band by the secondary user.

In Figure 1, we plot the energy efficiency at equilibrium as function of the SNR. Interestingly, we see that the energy efficiency of the primary user at the Stackelberg equilibrium performs the same than in the sensing scenario till $12 \mathrm{~dB}$ while the energy efficiency of the secondary user at the Stackelberg equilibrium is

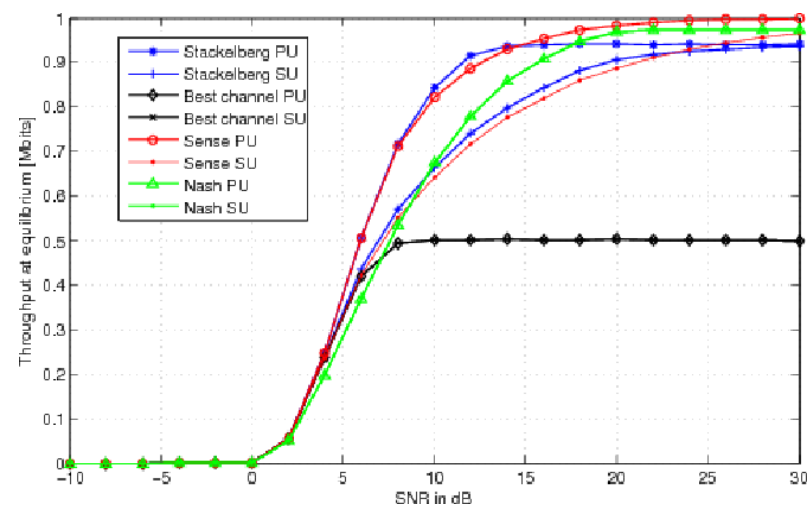

Fig. 2. Throughput at the equilibrium as function of the SNR for different schemes.

always the same than in the sensing scheme. Notice here that although not shown in Fig. 1, the PU's energy efficiency in the sensing scenario saturates at about 40 $\mathrm{dB}$. Moreover, the Stackelberg model outperforms all the other strategies. This due to the Stackelberg mechanism in which the primary user anticipates the secondary user's action. In particular, we found out that the primary user achieves an energy efficiency gain up to $80 \%$ with respect to the Nash strategy at $12 \mathrm{~dB}$. As expected, results in Fig. 1 also show that the energy efficiency for the secondary user at the Stackelberg equilibrium is less than the one obtained at Nash equilibrium. This is due to the fact that in Nash model, the PU does not anticipate the secondary user's action. Notice that, as the SNR decreases, all configurations tend towards having the same (zero) energy efficiency. This can be justified by the fact that, at low SNR regime, whatever the power control strategy each user chooses, the signal is overwhelmed by the noise.

Figure 2 depicts the throughput at the equilibrium. We observe approximately the same observations than in Fig. 1. Of particular interest is the fact that the PU still outperforms all the other strategies till $\mathrm{SNR}=15$ $\mathrm{dB}$ whereas the energy efficiency of the SU at the Stackelberg equilibrium is still less than the one obtained at the Nash equilibrium.

\section{B. Learning for equilibrium solutions}

To proceed further with the analysis, we resort to simulate how the PU and the SU users converge to the equilibria according to Algorithm 1 presented in Section IV. The noise signal variance is $\sigma^{2}=0.1$ which corresponds to a $\mathrm{SNR}=10 \mathrm{~dB}$.

In Figure 3, we plot the energy efficiency of the PU and the SU at the Nash equilibrium proposed in [2] 


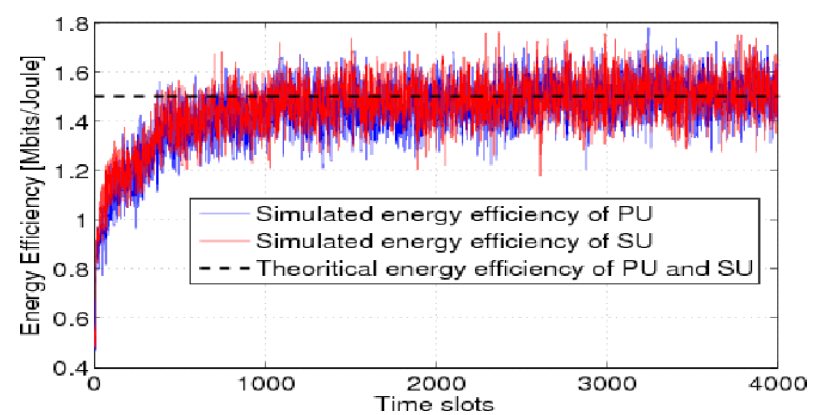

Fig. 3. The energy efficiency at the Nash equilibrium for both PU and SU.

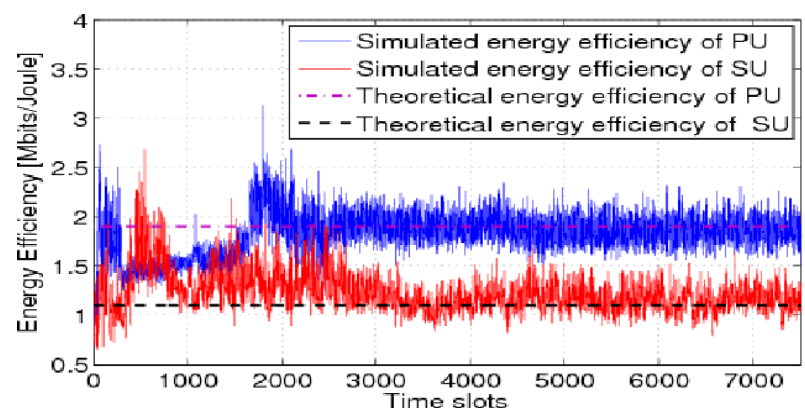

Fig. 4. The energy efficiency at the Stackelberg equilibrium for both PU and SU.

depending on time. It is clear that both the PU and the SU converge to the same energy efficiency since the Nash game is a one-shot game. We also observe that both the PU and the SU converge to exactly the same energy efficiency of $1.6 \mathrm{Mbit} / \mathrm{J}$ oule than the one obtained in Fig. 1 at $\mathrm{SNR}=10 \mathrm{~dB}$.

Next, we plot in Figure 4, the convergence of the energy efficiency at the Stackelberg equilibrium for both the PU and the SU. Again we observe that the PU and the SU converge to the same energy efficiency of 1.9 Mbit/Joule and of 1.1 Mbit/Joule respectively obtained in Fig. 1 at $\mathrm{SNR}=10 \mathrm{~dB}$. Moreover, as expected, that the energy efficiency at the Stackelberg equilibrium of the PU is higher than the energy efficiency of the SU.

Let us now focus on the coordination between the PU and the SU. We illustrate in Figure 5 the transmission power of the PU and the $\mathrm{SU}$ at the equilibrium on the first carrier on different time slots. We observe that the PU transmit on the first carrier during time slots $\{2,3,4,6,9\}$ whereas the $\mathrm{SU}$ transmits on the first carrier during time slots $\{1,5,7,8,10\}$. This result validates the coordination property claimed in Prop. 3.

\section{CONCLUSION}

We have analyzed in this paper the impact of hierarchically allocating transmit power in energy efficient

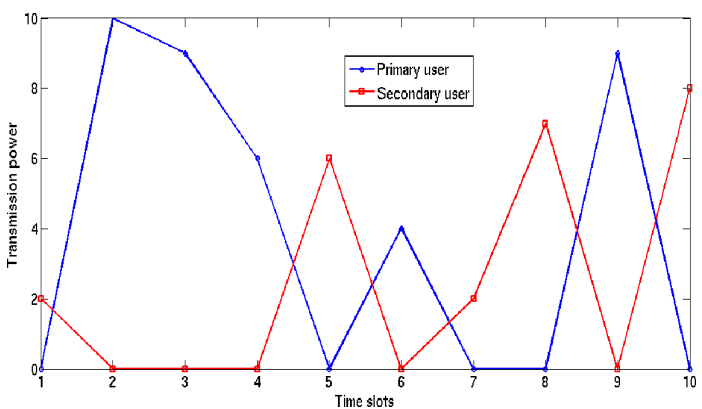

Fig. 5. The transmission power of the PU and the SU on the first carrier at the equilibrium.

cognitive radio networks. One important task here is that contrary to typical power control mechanisms, the secondary user has only to reliably detect the carrier used by the the primary user and not the primary user's transmit power as it is the case in the single carrier context in [8]). Simulation results validate our claims and offer insights into how much one can gain from the Stackelberg formulation in terms of energy efficiency. In particular, we have shown by means of a Stackelberg game model that the PU can optimize his individual energy efficiency while still maximizing his throughput.

\section{REFERENCES}

[1] Y. Hayel, M. Haddad, "A Stackelberg Approach for Energy Efficient Multicarrier Systems", in IEEE Globecom, 2012.

[2] F. Meshkati, M. Chiang, H. V. Poor and S. C. Schwartz, "A game-theoretic approach to energy-efficient power control in multi-carrier CDMA systems", IEEE Journal on Selected Areas in Communications, Vol. 24, No. 6, June 2006, pp. 1115-1129.

[3] S. Haykin, "Cognitive radio: brain-empowered wireless communications", IEEE Journal on Selec. Areas in Comm., Vol. 23, pp. 201-220, 2005.

[4] M. Haddad, A. Hayar and M. Debbah, "Spectral Efficiency of Spectrum Pooling Systems", IET Special Issue on Cognitive Spectrum Access, Vol. 2, No. 6, pp. 733-741, July 2008.

[5] M. Haddad, A. Menouni and G. E. Oien, "Downlink Distributed Binary Power Allocation for Cognitive Radio Networks", PIMRC 2008, Cannes, France, September 2008.

[6] D. J. Goodman and N. B. Mandayam, "Power Control for Wireless Data", IEEE Personal Communications, Vol. 7, No. 2, April 2000, pp. 48-54,

[7] V. Rodriguez, "An Analytical Foundation for Resource Management in Wireless Communication”, IEEE Globecom, 2003.

[8] S. Lasaulce, Y. Hayel, R. El Azouzi, and M. Debbah, "Introducing Hierachy in Energy Games", IEEE Transaction on Wireless Communication, vol. 8, no. 7, pp. 3833-3843, July 2009.

[9] H. Urkowitz, "Energy detection of unknown deterministic signals", Proceeding of the IEEE, Vol. 55, No. 4, pp. 523-531, Apr. 1967.

[10] Dandawat, A. V. and G. B. Giannakis, "Statistical tests for presence of cyclostationarity", IEEE Trans. Signal Processing, 42(9), pp. 2355-2369. 